\title{
Huge magnetoresistance and ultrasharp metamagnetic transition in polycrystalline $\mathrm{Sm}_{0.5} \mathrm{Ca}_{0.25} \mathrm{Sr}_{0.25} \mathrm{MnO}_{3}$
}

Sanjib Banik ${ }^{1}$, Kalipada Das ${ }^{1,2}$, Tapas Paramanik ${ }^{1,3}$, Niranjan Prasad Lalla ${ }^{4}$, Biswarup Satpati $\mathbb{C}^{5}$, Kalpataru Pradhan ${ }^{1}$ and Indranil Das ${ }^{1}$

\begin{abstract}
Large magnetoresistive materials are of immense interest for a number of spintronic applications, such as the development of high-density magnetic memory devices, magnetic sensors, and magnetic switches. Colossal magnetoresistance, in which the resistivity changes by several orders of magnitude $\left(\sim 10^{4} \%\right)$ in an external magnetic field, occurs mainly in phase-separated oxide materials, namely, manganites, owing to the phase competition between the ferromagnetic metallic and antiferromagnetic insulating regions. Can the magnetoresistance be further enhanced by tuning the volume fraction of the two phases? In this work, we report a huge colossal magnetoresistance along with an ultrasharp metamagnetic transition in a half-doped $\mathrm{Sm}_{0.5} \mathrm{Ca}_{0.25} \mathrm{Sr}_{0.25} \mathrm{MnO}_{3}$ manganite compound by suitably tuning the volume fraction of the competing phases. The obtained magnetoresistance value at $10 \mathrm{~K}$ is as large as $\sim 10^{13} \%$ in a $30 \mathrm{kOe}$ external magnetic field and $\sim 10^{15} \%$ in a $90 \mathrm{kOe}$ external magnetic field and is several orders of magnitude higher than any other observed magnetoresistance value reported thus far. Using model Hamiltonian calculations, we have shown that the inhomogeneous disorder, deduced from tunneling electron microscopy, suppresses the CE-type phase and seeds the ferromagnetic metal in an external magnetic field.
\end{abstract}

\section{Introduction}

For the last several years, the search for materials with large magnetoresistances (MRs) and studies on related phenomena have been in the forefront of worldwide research activity ${ }^{1-7}$ owing to their widespread application in the field of magnetic sensors, magnetic memory devices, magnetic switches, etc ${ }^{8-12}$. The highest value of MR in principle can be achieved if the resistivity of the material can be transformed from an extreme insulating material (such as mica) to a very good metallic material (such as copper) by applying a magnetic field.

Correspondence: Indranil Das (indranil.das@saha.ac.in) or

Kalpataru Pradhan (kalpataru.pradhan@saha.ac.in)

${ }^{1}$ CMP Division, Saha Institute of Nuclear Physics, HBNI, 1/AF-Bidhannagar, Kolkata 700 064, India

${ }^{2}$ Department of Physics, Seth Anandram Jaipuria College, 10-Raja Naba Krishna Street, Kolkata 700005, India

Full list of author information is available at the end of the article.
In perovskite manganite, an insulating state is observed in the charge-ordered antiferromagnetic (CO-AFM) sample, which generally appears near half doping. The critical magnetic field, which is required to destabilize the CO-AFM state, increases with decreasing bandwidth. The CO-AFM state can be weakened by introducing ferromagnetic proximity as follows: (i) by effectively increasing the bandwidth of the $e_{g}$ electrons via substitution of larger cations at the A-sites ${ }^{13-16}$, (ii) via B-site doping (e.g., Cr or Ru doping on Mn sites) ${ }^{17-19}$, and (iii) by making FM-AFM core-shell nanostructures (or nanoparticles) $)^{20-22}$. Thus, inducing a ferromagnetic phase fraction in an antiferromagnetic manganite or, in other words, engineering electronic phase separation is an effective tool to enhance the MR. Recently, a great amount of effort has been devoted to control the electronic phase separation in bulk and low dimensional manganites ${ }^{23-29}$. 
It is known that the CO-AFM state in a low bandwidth $\mathrm{Sm}_{0.5} \mathrm{Ca}_{0.5} \mathrm{MnO}_{3}$ (SCMO) is very stable ${ }^{30}$, and an $\sim 500 \mathrm{kOe}$ (at temperature $(T)=4.2 \mathrm{~K}$ ) critical magnetic field $\left(\mathrm{H}_{\mathrm{CR}}\right)$ is required to destabilize the $\mathrm{CO}$ state. On the other hand, CO-AFM and FM metallic phases coexist in $\mathrm{Sm}_{0.5} \mathrm{Sr}_{0.5} \mathrm{MnO}_{3}$ and are metallic at low temperatures $^{31,32}$. In our case, we prepared $\mathrm{Sm}_{0.5} \mathrm{Ca}_{0.25} \mathrm{Sr}_{0.25} \mathrm{MnO}_{3}$ (SCSMO) by replacing half of the $\mathrm{Ca}^{2+}$ ions with $\mathrm{Sr}^{2+}$ ions. This substitution reduces the charge-ordering temperature $\left(T_{\mathrm{CO}}\right)$ and Neel temperature $\left(T_{\mathrm{N}}\right)$ of SCSMO by $\sim 50 \mathrm{~K}$ compared with its parent compound $\mathrm{SCMO}^{14}$. Although SCSMO remains a strong insulator $\left(\sim 10^{12} \mathrm{Ohm}-\mathrm{cm}\right)$ at low temperature, the critical field decreases to $48 \mathrm{kOe}$ (for SCSMO) from $500 \mathrm{kOe}$ (for SCMO).

For the prepared polycrystalline SCSMO, surprisingly, we obtain an unprecedented MR of $\sim 10^{15} \%$ at $10 \mathrm{~K}$ upon application of a $90 \mathrm{kOe}$ magnetic field. This MR value in the bulk SCSMO sample is undoubtedly a record value compared with the previously observed MR in any chargeordered compounds. For example, the MR value for the $\mathrm{La}_{0.5} \mathrm{Ca}_{0.5} \mathrm{MnO}_{3}$ compound is $10^{8} \%$ at $57 \mathrm{~K}$ for an $80 \mathrm{kOe}$ applied field. Via a substrate-induced strain, a huge MR of $10^{11} \%$ has also been observed in $\mathrm{Nd}_{0.5} \mathrm{Ca}_{0.5} \mathrm{MnO}_{3}$ thin film at $50 \mathrm{~K}$ upon application of a $70 \mathrm{kOe}$ magnetic field ${ }^{33}$. The charge-ordered $\operatorname{Pr}_{0.5} \mathrm{Ca}_{0.5} \mathrm{MnO}_{3}$ compound shows an MR of $10^{8} \%$ at $60 \mathrm{~K}$ upon application of a $150 \mathrm{kOe}$ magnetic field owing to the strain induced by the substrate ${ }^{34}$. Moreover, a thin film made of our parent compound SCMO shows an MR of $10^{6} \%$ upon application of a $200 \mathrm{kOe}$ external field at $57 \mathrm{~K}^{35}$. In contrast, the MR value of our SCSMO sample reaches $10^{6} \%$ at $50 \mathrm{~K}$ in an only $30 \mathrm{kOe}$ magnetic field.

We also find an ultrasharp metamagnetic transition ${ }^{36-39}$ below $10 \mathrm{~K}$. We explain the metamagnetic as well as ultrasharp metamagnetic transition using a martensitic scenario. Our Monte Carlo simulations using a two-band double-exchange model show that A-site disorder suppresses the CE-type phase, but the system remains insulating without any external magnetic field. In an external magnetic field, the inhomogeneous disorder seeds the ferromagnetic metallic clusters into the system, and as a result, resistivity decreases at low temperatures, which gives rise to a large MR.

\section{Materials and methods Sample preparation}

The polycrystalline SCSMO compound was prepared via the well-known sol-gel chemistry route. For the sample preparation, high purity $(99.99 \%) \mathrm{Sm}_{2} \mathrm{O}_{3}, \mathrm{CaCO}_{3}, \mathrm{Sr}\left(\mathrm{NO}_{3}\right)_{2}$, $\mathrm{MnO} 2$, oxalic acid, and citric acid were used as the constituents. The appropriate amounts of $\mathrm{Sm}_{2} \mathrm{O}_{3}$ and $\mathrm{CaCO}_{3}$ were converted to their nitrate forms using concentrated nitric acid $\left(\mathrm{HNO}_{3}\right)$ and dissolved into Millipore water. Because $\mathrm{MnO}_{2}$ does not dissolve directly in $\mathrm{HNO}_{3}$, the appropriate amount of oxalic acid was used to convert it into the oxalate form, which easily dissolves in Millipore water in the presence of nitric acid. After preparation of the individual clear solutions of the constituent elements $\left(\mathrm{Sm}_{2} \mathrm{O}_{3}, \mathrm{CaCO}_{3}, \mathrm{Sr}\left(\mathrm{NO}_{3}\right)_{2}\right.$, and $\left.\mathrm{MnO}_{2}\right)$, all solutions were homogenously mixed by using a magnetic stirrer for 30 minutes. To make the precursor solution, a suitable amount of citric acid was added to this homogenous solution. Extra water was slowly evaporated by continuously heating this solution using a water bath while maintaining the temperature of the water bath at $\sim 80{ }^{\circ} \mathrm{C}$. This slow evaporation continued until gel formation. After the gel formed, it decomposed at a slightly higher temperature $\left(\sim 150{ }^{\circ} \mathrm{C}\right)$, and a black porous powder was formed. To evaporate the extra citric acid, porous powder was directly heated at $500{ }^{\circ} \mathrm{C}$ for $4 \mathrm{~h}$. To obtain crystalline single-phase bulk compounds, the powder sample was first pelletized and heated at $1300{ }^{\circ} \mathrm{C}$ for the time period of $\sim 36 \mathrm{~h}$.

\section{TEM measurements}

High-resolution transmission electron microscopy (HRTEM) was performed using a FEI Tecnai, F30 microscope with a point-to-point resolution of $1.8 \AA$. The composition of the prepared sample was determined via energy dispersive X-ray spectroscopy (EDS) analysis using an analyzer mounted on the same microscope.

\section{Electrical transport measurement}

Resistance measurements in the absence of an external magnetic field were carried out using a Keithley source and measure unit $2651 \mathrm{~A}$ via the four probe method in the temperature range of $20 \mathrm{~K}$ to $300 \mathrm{~K}$. The low temperature, measurement of the zero field resistivity was extended below $20 \mathrm{~K}$ using a Keithley Electrometer $6517 \mathrm{~A}$ in the capacitor arrangement method, and below $10 \mathrm{~K}$, the resistance value exceeded our measurement limit-I $\left(10^{13} \mathrm{ohm}\right)$. In-field resistivity measurements were carried out using the 2651 A with the measurement limit-II of $10^{11} \mathrm{ohm}$.

\section{Magnetic property measurements}

Magnetic properties were measured using a superconducting quantum interference device magnetometer (SQUID-VSM) from Quantum Design in the temperature range of $2-300 \mathrm{~K}$ with a maximum magnetic field value of $70 \mathrm{kOe}$.

\section{XRD measurements}

Room temperature powder X-ray diffraction (XRD) and temperature-dependent XRD measurements in the temperature range of $15-300 \mathrm{~K}$ were performed using a RIGAKU-TTRAX-III diffractometer with a rotating anode $\mathrm{Cu}$ source of wavelength $\lambda=1.54 \AA\left(\mathrm{CuK}_{\alpha}\right)$. Measurements were performed at a $9 \mathrm{~kW}$ power. To observe the effect of the magnetic field on the XRD linewidth 
broadening, zero field and $70 \mathrm{kOe}$ in-field followed by zero field measurements were performed using the low temperature and high magnetic field XRD setup at UGCDAE CSR (Indore).

\section{Heat capacity measurements}

The magnetic field dependence as well as temperature dependence of the heat capacity of the sample was determined using the physical property measurements system from Quantum Design.

\section{Results and discussion}

\section{Synthesis and structural characterization}

High-quality polycrystalline SCSMO was prepared via the well-known sol-gel technique (see the methods for details). The crystalline structure and single-phase nature of the sample were studied using room temperature XRD and transmission electron microscopy (TEM). The XRD pattern (see Fig. 1a) (analysis is discussed in supplementary section I(A)) and the TEM diffraction pattern (see Fig. 1e) show the orthorhombic crystallographic symmetry (Pnma) of the sample. Room temperature chemical analysis using EDS measurement (see supplementary section $\mathrm{I}(\mathrm{C})$ ) shows that the elements are distributed homogeneously and stoichiometrically.

We also present XRD analysis at several low temperatures in Fig. 1b-d. With lowering of the temperature, another crystallographic phase with monoclinic symmetry P21/m along with the room temperature symmetry Pnma, i.e., combinations of Pnma and P21/m, appears below $T \leq$ $120 \mathrm{~K}$. The evolution of the new peak corresponding to the $\mathrm{P} 21 / \mathrm{m}$ monoclinic space group is shown in Fig. 1c. This figure indicates that below $120 \mathrm{~K}$, the new peak appears at $2 \theta=47.5^{\circ}$, and its intensity remains almost constant below $100 \mathrm{~K}$. This implies that below $100 \mathrm{~K}$, the P21/m phase becomes trapped within the host Pnma phase, creating a lattice strain in the host Pnma phase. Strain is also evident from the TEM analysis (see Fig. 1f) at $100 \mathrm{~K}$. It was previously shown that the antiferromagnetic transition is associated with structural transition from the orthorhombic to orthorhombic + monoclinic phase at $135 \mathrm{~K}$ in $\mathrm{Sm}_{0.5} \mathrm{Sr}_{0.5} \mathrm{MnO}_{3}{ }^{40,41}$. Therefore, the monoclinic structure favors the antiferromagnetic phase in $\mathrm{Sm}_{0.5} \mathrm{Sr}_{0.5} \mathrm{MnO}_{3}$. In our case, the

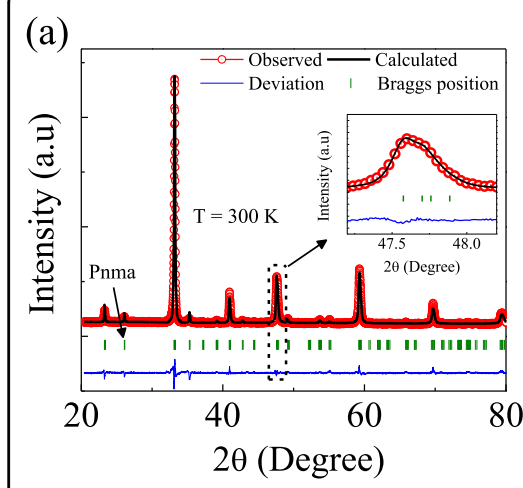

(b)

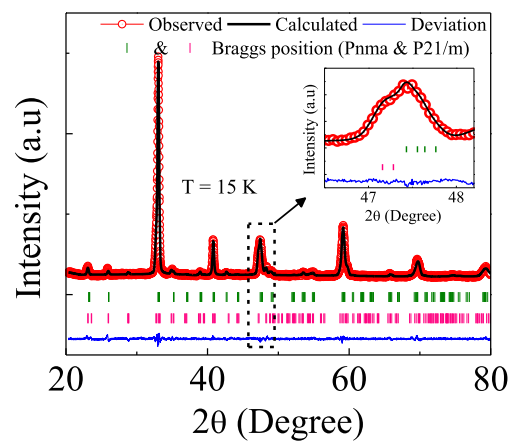

(c)

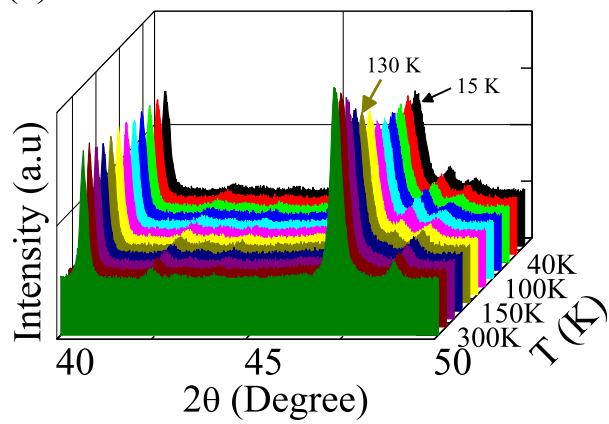

(d)

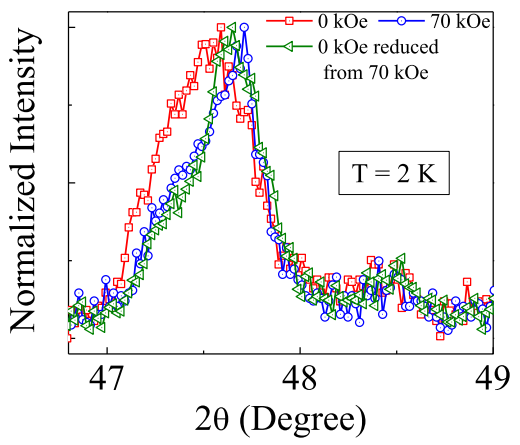

(e)

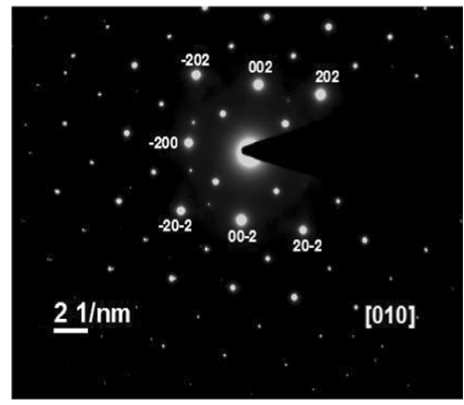

(f)

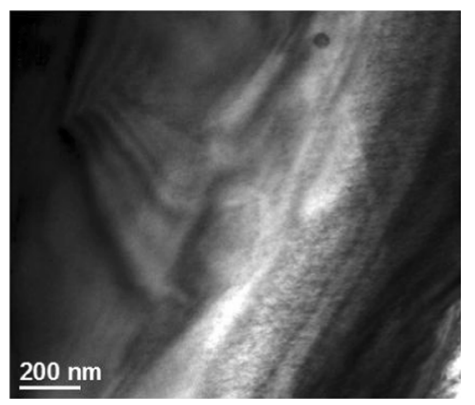

Fig. 1 XRD and TEM analysis $\mathbf{a}$, $\mathbf{b}$ show the profile fitting of the room temperature (RT) and low temperature (15 K) XRD data using Pnma and $(\mathrm{Pnma}+\mathrm{P} 21 / \mathrm{m})$ space groups. The insets of $\mathbf{a}$ and $\mathbf{b}$ show the fitting of the peaks at $2 \theta=47.5^{\circ}$, where a new monoclinic phase (P21/m) appears at low temperature. $\mathbf{c}$ displays the evolution of the new peak of the P21/m space group with temperature. $\mathbf{d}$ shows the XRD linewidth modification in the presence of a zero magnetic field and a $70 \mathrm{kOe}$ magnetic field and after removing the field. e displays typical (100) zone axis ED patterns at room temperature. The pattern was indexed using an orthorhombic structure (Pnma). $\mathbf{f}$ demonstrates a (001) bright-field image recorded at $100 \mathrm{~K}$ during TEM measurements. For HRTEM analysis, please see Supplementary section I(B) 
SCSMO compound also undergoes the same kind of structural transition at $120 \mathrm{~K}$. We will show in the next section that the system also undergoes an antiferromagnetic transition at $120 \mathrm{~K}$. Thus, the monoclinic P21/m phase (shown in Fig. 1b, c) is associated with the CE-AFM phase.

The effect of the external magnetic field $(70 \mathrm{kOe})$ on the XRD linewidth broadening at $2 \mathrm{~K}$ (see Fig. 1d) indicates a decrease in the linewidth (full width at half maxima) at $2 \theta=47.5^{\circ}$ from $0.58^{\circ}$ to $0.46^{\circ}$, which then remains at the same value even after removing the field. This shows that strain decreases in an external magnetic field and then remains as it is even after removing the field.

\section{Magnetic and magnetotransport measurements}

The temperature-dependent magnetization and resistivity for different applied magnetic fields are shown in Fig. 2a, b. Interestingly, below $60 \mathrm{~K}$, magnetization increases, but the ferromagnetic fraction is very small for the 100 Oe magnetic field, as confirmed by the thermoremanent magnetization measurements (discussed in supplementary section I(D)). As a result, the system without any magnetic field remains insulating at low temperature, as shown in Fig. $2 \mathrm{~b}$, but the stability of the CO-AFM state decreases (discussed in the next paragraph). This is unlike our previously studied core-shell (ferromagnetic core and antiferromagnetic shell) nanostructures ${ }^{22}$, for which a comparatively small resistivity $\left(\sim 10^{2} \mathrm{Ohm}-\mathrm{cm}\right)$ was obtained at low temperatures. Below a certain temperature $(T<10 \mathrm{~K})$, the value of the resistance is $R \sim 10^{13}$ ohm for SCSMO, which is the limiting value of our measuring instruments (see the methods section for details). The ordering temperatures ( $T_{\mathrm{CO}}$ and $\mathrm{T}_{\mathrm{N}}$ ) are indicated in the inset of Fig. 2a, b, and a similar $T_{\mathrm{CO}}$ is also obtained from the heat capacity measurement (please see supplementary section I(E)).

A $30 \mathrm{kOe}$ magnetic field induces a larger ferromagnetic fraction, and as a result, the resistivity decreases considerably (from $10^{13} \mathrm{Ohm}-\mathrm{cm}$ to $\rho \sim 17.5 \mathrm{Ohm}-\mathrm{cm}$ at $T=$ $2.5 \mathrm{~K}$ ) at low temperatures (see Fig. 2a, b). This shows not only that $\mathrm{T}_{\mathrm{CO}}$ decreases but also that the robustness of the CO-AFM state is reduced in SCSMO. A huge hysteresis is observed between the field cooling and heating cycles for $30 \mathrm{kOe}$ in the temperature range of $50 \mathrm{~K}<T<100 \mathrm{~K}$ in both the magnetization and resistivity curves, which is the signature of field-induced electronic phase separation ${ }^{42}$.
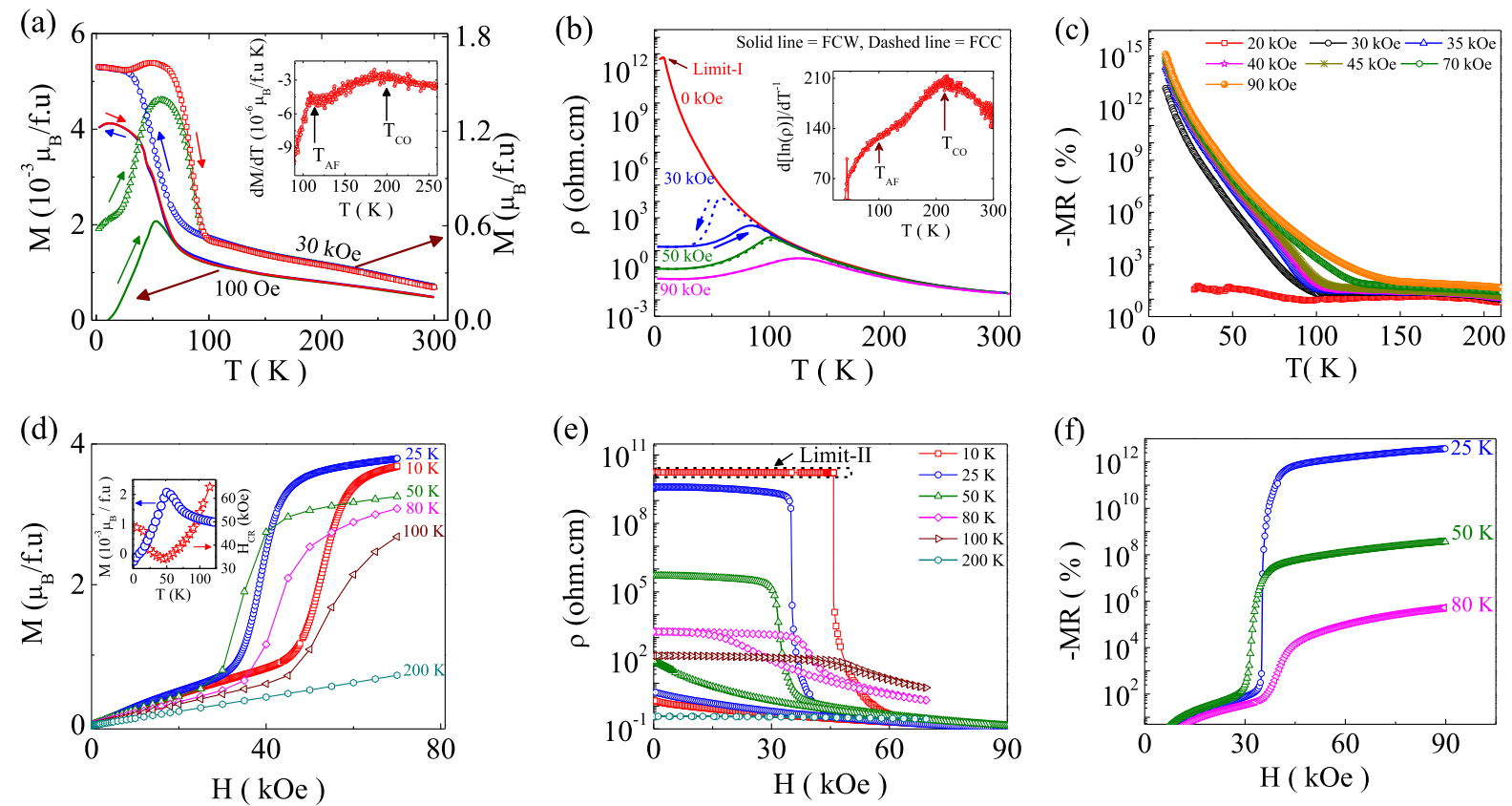

Fig. 2 Magnetotransport properties a Variation of magnetization with temperature in $100 \mathrm{Oe}$ and $30 \mathrm{kOe}$ external magnetic fields. The inset shows the signature of $\mathrm{CO}$ and AFM ordering, indicated by arrows, from the temperature derivative of M(T) data taken in the field cooled warming (FCW) protocol in the presence of a 100 Oe magnetic field. b Temperature-dependent resistivity without (red) and with (30 kOe (blue), $50 \mathrm{kOe}$ (olive) and $90 \mathrm{kOe}$ (violet)) external magnetic fields. The dotted lines represent the resistivity data taken during the field cooling cycle, and the solid lines represent the FCW cycle. The inset shows the variation of the activation energy $\left(E_{A} \infty \mathrm{d}[\ln (\rho)] / \mathrm{dT}^{-1}\right)$ with temperature, calculated from the temperature dependence of the zero field resistivity data, and the ordering temperatures are indicated by arrows. c Magnetic field dependence of MR on temperature for different magnetic fields. $\mathbf{d}$ Magnetization vs. magnetic field and e resistivity vs. magnetic field at different temperatures. The inset in $\mathbf{d}$ shows the temperature dependence of $\mathrm{H}_{C R}$ and zero field cooled (ZFC) magnetization in a 100 Oe magnetic field. $\mathbf{f}$ Magnetic fielddependent magnetoresistance at three different temperatures. See the materials and methods section for an explanation of limit-I and limit-II, mentioned in $\mathbf{b}$ and $\mathbf{e}$, respectively 

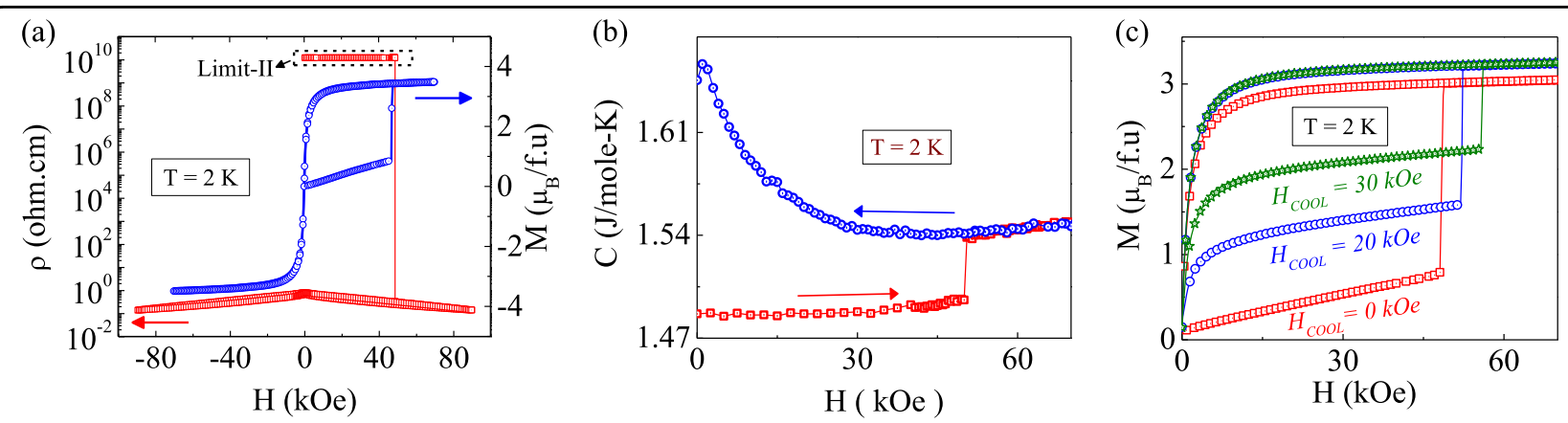

Fig. 3 Ultrasharp metamagnetic transition a Magnetization (and resistivity) vs. magnetic field at $2 \mathrm{~K}$. See the Materials and methods section for an explanation of limit-II. b Variation of heat capacity with an external magnetic field at $2 \mathrm{~K}$. Here, the red and blue symbols represent the $\mathrm{C}(\mathrm{H})$ data taken during increasing and decreasing fields, respectively. c Effect on isothermal magnetization of different cooling fields at $2 \mathrm{~K}$

With a further increase in the applied magnetic field, this coexistence is suppressed, and the low temperature resistivity goes to $10^{-2} \mathrm{Ohm}-\mathrm{cm}$ (for $90 \mathrm{kOe}$ ).

To quantify the field-induced change in resistivity, we plot the MR $(=(\rho(\mathrm{H})-\rho(0)) / \rho(\mathrm{H}) \times 100)$ vs. temperature at different magnetic fields in Fig. 2c. MR is $~ 10 \%$ for 20 kOe but astonishingly increases to $10^{13} \%$ for a $30 \mathrm{kOe}$ external magnetic field and to as large as $\sim 10{ }^{15} \%$ at $10 \mathrm{~K}$ for a $90 \mathrm{kOe}$ magnetic field. The MR value as high as $10^{15} \%$ in the polycrystalline stable SCSMO compound is a unique observation.

The isothermal magnetization measured in the zero field cooled (ZFC) protocol at different temperatures (presented in Fig. 2d) shows the field-induced metamagnetic transitions. The experimentally obtained saturation magnetic moment $\left(3.82 \mu_{\mathrm{B}}\right)$ at $30 \mathrm{~K}$ is close to the estimated magnetic moment for the full saturation of the $\mathrm{Mn}^{3+} / \mathrm{Mn}^{4+}$ and $\mathrm{Sm}^{3+}$ ions $\left(3.85 \mu_{\mathrm{B}}\right)$. This clearly indicates that the CO-AFM state transforms completely upon application of the magnetic field via the metamagnetic transition near the critical magnetic field $\mathrm{H}_{\mathrm{CR}}$. The temperature variation of $\mathrm{H}_{\mathrm{CR}}$ measured from $\mathrm{M}(\mathrm{H})$ isotherms is opposite to that of ZFC magnetization, as shown in the inset of Fig. 2d. The FM components present in the ZFC sample act as nucleation centers and grow at the expense of AFM components in an external magnetic field, resulting in a metamagnetic transition at $\mathrm{H}_{\mathrm{CR}}$. As the $\mathrm{ZFC}$ magnetization increases from $2 \mathrm{~K}$ to $50 \mathrm{~K}$, the enhanced thermal energy reduces $\mathrm{H}_{\mathrm{CR}}$ from $47.8 \mathrm{kOe}$ at $2 \mathrm{~K}$ to 34.5 $\mathrm{kOe}$ at $50 \mathrm{~K}$. For $\mathrm{T}>50 \mathrm{~K}, \mathrm{H}_{\mathrm{CR}}$ increases owing to reduction of the ZFC magnetization. In the absence of AFM components, above $T_{N}$, the field-induced metamagnetic transition vanishes.

Figure $2 \mathrm{e}$ shows the resistivity vs. magnetic field isotherms measured at different temperatures. Interestingly, the isotherms measured at lower temperature $(10 \mathrm{~K})$ show an exceptionally sharp metamagnetic jump. With increasing temperature, the loop area between increasing and decreasing field sweeps decreases, and at $100 \mathrm{~K}$, it almost disappears. Thus, we believe that field-induced phase coexistence is sustained up to $\sim 120 \mathrm{~K}$, which is the antiferromagnetic ordering temperature. Another point to note here is that the system remains in the low resistivity state even after removing the field for $T<50 \mathrm{~K}$. Owing to the resistivity measurement limitation, the resistivity is limited to $10^{10} \mathrm{ohm}-\mathrm{cm}$ in an external magnetic field, and as a result, we can only measure the MR for temperatures above $25 \mathrm{~K}$. The MR is equal to $10^{12} \%\left(10^{4} \%\right)$ at $25 \mathrm{~K}$ (80 K) for a 45 kOe magnetic field, as shown in Fig. 2f, and will be even larger for lower temperatures.

\section{Ultrasharp metamagnetic transition}

Interestingly, the resistivity (and magnetization) vs. magnetic field isotherms (see Fig. 3a) at $2 \mathrm{~K}$ show exceptionally ultrasharp steps with a width of $\sim 10$ Oe (the smallest step used during the measurement is $3 \mathrm{Oe}$ ). To explore the origin of this ultrasharp metamagnetic transition, we now measure the heat capacity $(C)$ with a magnetic field. During the field increasing cycle, the heat capacity data show a step-like behavior at $\sim 48 \mathrm{kOe}$, as shown in Fig. 3b, which has a one-to-one correspondence with the step-like behavior observed in the magnetization and resistivity isotherms. This is opposite to the case observed in earlier studies where $C$ decreased sharply with $\mathrm{H}$ at the transition point, which was associated with the rise in temperature of the system owing to the release of energy that assisted the abrupt field-induced transi$\operatorname{tion}^{36,43}$. The sharp increase in the heat capacity at the 48 kOe magnetic field rules out the possibility of an exothermic temperature-driven avalanche metamagnetic transition being observed in our system, and we believe that the system changes martensitically.

To ascertain the martensitic nature, we also studied the isothermal magnetization (at $2 \mathrm{~K}$ ) for different cooling fields 9see Fig. 3c). With increasing cooling field, the FM fraction increases, and as a result, the interfacial elastic energy increases. Owing to this, the critical field increases with increasing cooling field. At the same time, the critical 
field decreases slightly when we increase the sweep rate from $10 \mathrm{Oe} / \mathrm{sec}$ to $200 \mathrm{Oe} / \mathrm{sec}$ for the ZFC sample (see supplementary section $\mathrm{I}(\mathrm{F})$ for details). This is because the lattice has adequate time to accommodate the induced interfacial strain between the AF and FM domains at a lower sweep rate, and a larger magnetic field is required to break the energy barrier. Both of these scenarios indicate the martensitic nature of the transition ${ }^{43-47}$. Our resistivity relaxation study (see supplementary section $\mathrm{I}(\mathrm{G})$ ) also confirms the martensitic nature of the metamagnetic transitions (Fig. 2d, e) at higher temperatures.

\section{Theoretical simulation}

In this section, we discuss the physical origin of the huge MR using a two-band double-exchange model including superexchange $(J)$ and electron-phonon coupling $(\lambda)$. Our model Hamiltonian ${ }^{19,48-51}$ (see Supplementary Section II for details), effectively a lattice of $\mathrm{Mn}$ ions, qualitatively reproduces the phase diagram of manganites. For SCMO (and SSMO)-like materials, involving two A-type elements ${ }^{30}$, one generally adds $\sum_{i} \varepsilon_{i} n_{i}$ such that $\overline{\varepsilon_{i}}=0$ to model the A-site cationic disorder ${ }^{48,51}$. Considering the fact that the $\mathrm{Sr}^{2+}$ ions randomly occupy the A-sites in the polycrystalline SCSMO compound and are larger in size compared with both $\mathrm{Sm}^{3+}$ and $\mathrm{Ca}^{2+}$, chemical disorder is created. This is also evident from the HRTEM images at room temperature, shown in Supplementary Section I(B) (Fig. 1b, c). Therefore, to model SCSMO, we neglect the disorder between $\mathrm{Sm}$ and $\mathrm{Ca}$ elements and incorporate the $\mathrm{Sr}$ disorder by adding $\sum_{i} \varepsilon_{i} n_{i}$ at each $\mathrm{Mn}$ site selected from the distribution $P\left(\varepsilon_{i}\right)=\frac{1}{4} \delta\left(\varepsilon_{i}-\Delta\right)+\frac{3}{4} \delta\left(\varepsilon_{i}+\Delta\right)$, where $\Delta$ is the quenched disorder potential. We add a Zeeman coupling term $\sum h \cdot S_{i}$ to the Hamiltonian in an external magnetic field, where $S_{\mathrm{i}}$ are $\mathrm{Mn} \mathrm{t}_{2 \mathrm{~g}}$ spins, to analyze the MR. We measure $J, \lambda, \Delta, h$, and temperature $(T)$ in units of kinetic hopping parameter $t$. The estimated value of $\mathrm{t}$ in manganites is $0.2 \mathrm{eV}^{48}$.

A spin-fermion Monte Carlo technique based on the traveling cluster approximation ${ }^{52}$ is used on a twodimensional $24 \times 24$ lattice (see supplementary section II for details). We use $J / t=0.1$ and $\lambda / t=1.65$, which reproduces the CE-CO-OO-I phase ${ }^{19}$ at an electron density $n=1-x=0.5$. The electron density is the number of itinerant $e_{g}$ electrons per $\mathrm{Mn}$ site in our calculations. The system, as shown in Fig. 4a, b, remains insulating at low temperatures for $\Delta / t=0.3$ and $h=0$, and the ferromagnetic structure factor $\mathrm{S}(0,0)$ is $\sim 0.001$ (for an outline of the resistivity and the magnetic structure factor calculations, please see Supplementary Section II).

The resistivity at low temperatures decreases with magnetic field $h$ (Fig. 4a), similar to our experimental results. This is owing to the increase of the FM correlations at low temperatures (Fig. 4b). On the other hand, SCMO-like materials (Fig. 4c) remain insulating at all temperatures, even for $h=0.03$. Recall that SCMO (SSMO) has a smaller (larger) bandwidth than SCSMO. In our model calculations, a larger $\lambda / t$ (and $J / t$ ) corresponds to a smaller bandwidth, or vice versa. For clarity, we use $\Delta / t=0$ (owing to the small mismatch between the Sm and Ca ionic radii) and set $\lambda / t=1.73$ and $J / t=$ 0.105 for SCMO-like materials. For SSMO-like materials, we set binary disorder ${ }^{48,51}$ with $\Delta / t=0.3$, and use $\lambda /$ $t=1.57$ and $J / t=0.095$. The FM correlations at low temperatures increase, and the resistivity decreases from SCMO-like to SCSMO-like to SSMO-like materials (Fig. 4d), which is qualitatively similar to the experimental results ${ }^{30-32}$.

We turn now to understand the MR in SCSMO by plotting Monte Carlo snapshots obtained at $T=0.01$. For $h=0.002$, the system remains insulating (see Fig. $4 \mathrm{~d}$ ) owing to the CE-type correlations without any significant ferromagnetic charge-disordered regions (see Fig. 5a, c) in the system. For $h=0.02$, ferromagnetic clusters coexist with zig-zag ferromagnetic chains, and the electron density is roughly homogeneous $(\sim 0.65)$ within the ferromagnetic clusters (see Fig. 5b, d). Moreover, the ferromagnetic clusters in SCSMO become connected with each other at reasonably large magnetic fields, unlike SCMO-like materials, for which the resistivity decreases
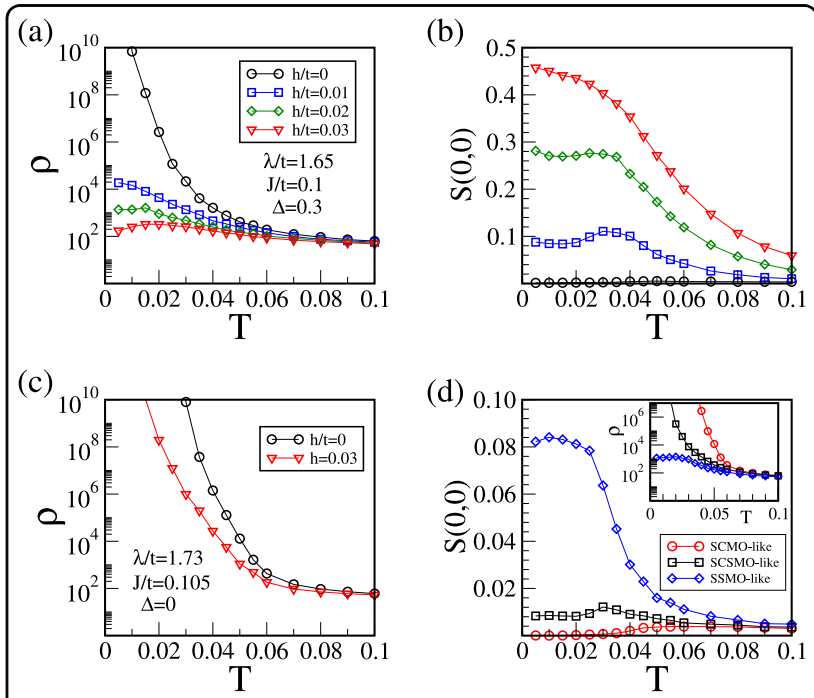

Fig. 4 Monte Carlo results Temperature dependence of $\mathbf{a}$ the resistivity $\rho$ in units of ha/ $/ \Pi^{2} e^{2}$ and $\mathbf{b}$ the FM structure factor $S(0,0)$ at different external magnetic field $h / t$ values for $\lambda / t=1.65, \mathrm{~J} / t=0.1$, and $\Delta / t=0.3$ (SCSMO-like materials). The legends in $\mathbf{a}, \mathbf{b}$ are the same. The electron density is fixed at $n=0.5$ in all figures. c Temperature dependence of $\rho$ for $\lambda / t=1.73, J / t=0.105$, and $\Delta / t=0$ (SCMO-like materials). $\mathbf{d}$ Temperature dependence of the FM structure factor $\mathrm{S}$ $(0,0)$ (inset: resistivity) for three sets of parameters (mimicking SCMO, SCSMO, and SSMO) in a very small magnetic field $h=0.002$. See the text for details 


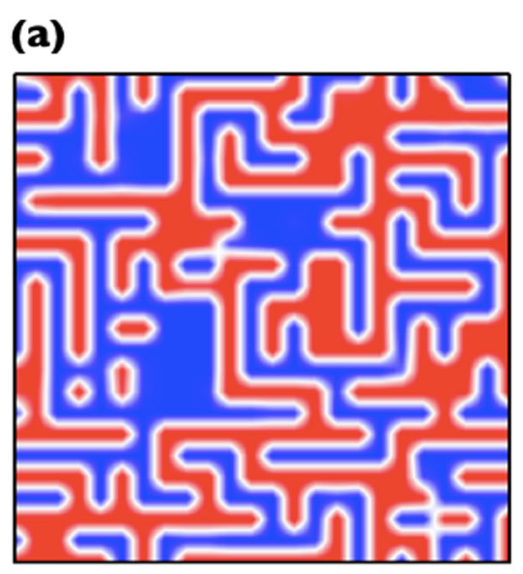

(b)

(c)

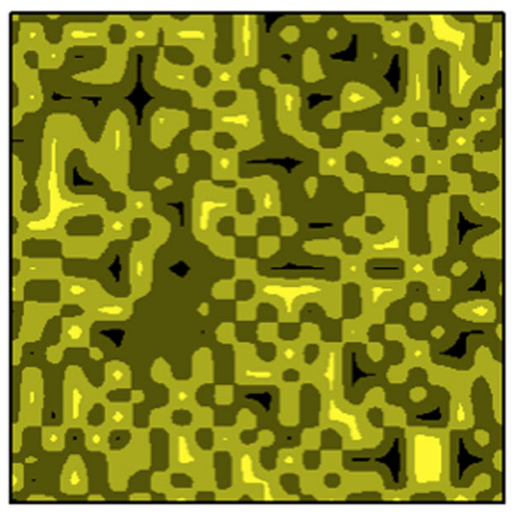

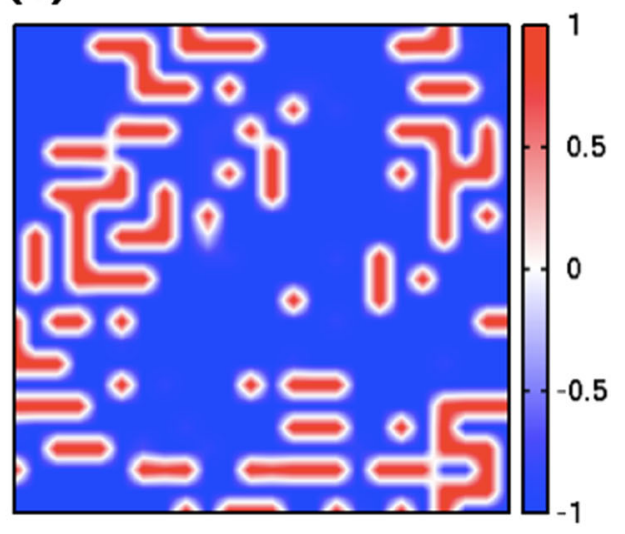

(d)

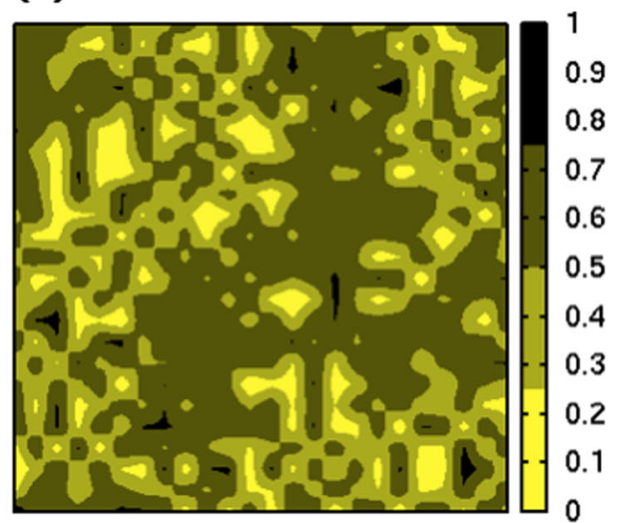

Fig. 5 Monte Carlo snapshots $\mathbf{a}, \mathbf{b}$ The $z$ components of simulated $\mathrm{Mn}\left(\mathrm{t}_{2 \mathrm{~g}}\right)$ spins. $\mathbf{c}$, $\mathbf{d}$ The electron density for each site on a $24 \times 24$ lattice at $T=$ 0.01 using $\lambda / t=1.65, \mathrm{~J} / t=0.1$, and $\Delta / t=0.3$. In $\mathbf{a}, \mathbf{c} h / t=0.002$, and in $\mathbf{b}$, $\mathbf{d} h / t=0.02$.

at lower temperatures. Therefore, overall, the disorder due to $\mathrm{Sr}$ ions present in SCSMO weakens the CE-type state of SCMO and seeds the ferromagnetic chargedisordered clusters, but the system remains insulating at low temperatures. In an external magnetic field, ferromagnetic clusters grow and become connected, giving rise to a large MR in SCSMO samples.

\section{Conclusion}

In summary, by controlling electronic phase separation in narrow bandwidth CO-AFM material, a record MR value $\left(\sim 10^{13} \%\right.$ in a $30 \mathrm{kOe}$ external magnetic field and $\sim 10^{15} \%$ in a $90 \mathrm{kOe}$ external magnetic field at $10 \mathrm{~K})$ till date is obtained in an SCSMO polycrystalline compound. The observed huge MR value has been explained by model Hamiltonian calculations. We also observed a magnetic field-induced ultrasharp metamagnetic transition at low temperatures. The many orders of magnitude higher MR than any other magnetoresistive materials reported thus far will motivate experimenters to design efficient magnetic materials for future applications.

\section{Acknowledgements}

Sanjib Banik acknowledges the Department of Atomic Energy (DAE), Govt. of India for financial support. K.P. acknowledges the use of the TCMP computer cluster at SINP. We sincerely thank P.B. Pal and M.G. Mustafa of SINP for useful discussions. The work at SINP has been supported through the CMPID-DAE project.

\section{Author details}

${ }^{1}$ CMP Division, Saha Institute of Nuclear Physics, HBNI, 1/AF-Bidhannagar, Kolkata 700 064, India. ${ }^{2}$ Department of Physics, Seth Anandram Jaipuria College, 10-Raja Naba Krishna Street, Kolkata 700005, India. Indian Institute of Technology, Kharagpur 721302, India. ${ }^{4}$ UGC-DAE Consortium for Scientific Research, University Campus, Khandwa Road, Indore 452017, India. ${ }^{5}$ Surface Physics and Material Science Division, Saha Institute of Nuclear Physics, HBNI, 1/AF-Bidhannagar, Kolkata 700 064, India

\section{Author contributions}

I.D. developed the concept of the study. S.B. and K.D. prepared the samples. S.B., K.D. and I.D. performed the experimental work and conducted the data analysis. N.P.L. did the field-dependent XRD measurements, B.S. carried out the TEM measurements, and K.P. performed the numerical calculations. S.B., K.D., T.P., K.P. and I.D. wrote the draft of the paper, and all authors reviewed the manuscript.

\section{Conflict of interest}

The authors declare that they have no conflict of interest. 


\section{Publisher's note}

Springer Nature remains neutral with regard to jurisdictional claims in published maps and institutional affiliations.

Supplementary Information is available for this paper at https://doi.org/ 10.1038/s41427-018-0085-7.

Received: 28 February 2018 Revised: 6 June 2018 Accepted: 13 June 2018. Published online: 20 September 2018

\section{References}

1. Moritomo, Y., Asamitsu, A., Kuwahara, H. \& Tokura, Y. Giant magnetoresistance of manganese oxides with a layered perovskite structure. Nature $\mathbf{3 8 0}, 141-144$ (1996).

2. Shimakawa, Y., Kubo, Y. \& Manako, T. Giant magnetoresistance in $\mathrm{Ti}_{2} \mathrm{Mn}_{2} \mathrm{O}_{7}$ with the pyrochlore structure. Nature 379, 53-55 (1996).

3. Uehara, M., Mori, S., Chen, C. H. \& Cheong, S. W. Percolative phase separation underlies colossal magnetoresistance in mixed-valent manganites. Nature 399 560-563 (1999)

4. Siwach, P. K., Singh, H. \& Srivastava, O. N. Low field magnetotransport in manganites. J. Phys. Condens. Matter 20, 273201 (2008).

5. Ali, M. N. et al. Large, non-saturating magnetoresistance in $\mathrm{WTe}_{2}$. Nature $\mathbf{5 1 4}$ 205-208 (2014)

6. Tafti, F. F., Gibson, Q. D., Kushwaha, S. K., Haldolaarachchige, N. \& Cava, R. J. Resistivity plateau and extreme magnetoresistance in LaSb. Nat. Phys. 12 272-277 (2016).

7. Baldini, M. et al. Origin of colossal magnetoresistance in $\mathrm{LaMnO}_{3}$ manganite. Proc. Natl Acad. Sci. USA 112, 10869-10872 (2015).

8. Asamitsu, A., Tomioka, Y., Kuwahara, H. \& Tokura, Y. Current switching of resistive states in magnetoresistive manganites. Nature 388, 50-52 (1997).

9. Bibes, M. \& Bathelemy, A. Oxide spintronics. IEEE Trans. Electron Devices 54, 1003-1023 (2007).

10. Sawa, A. Resistive switching in transition metal oxides. Mater. Today 11, 28-36 (2008).

11. Rubi, D. et al. Two resistive switching regimes in thin film manganite memory devices on silicon. Appl. Phys. Lett. 103, 163506 (2013).

12. Hoffman, J. D., Wu, S. M., Kirby, B. J. \& Bhattacharya, A. Tunable noncollinear antiferromagnetic resistive memory through oxide superlattice deasign. Phys. Rev. Appl. 9, 044041 (2018).

13. Arulraj, A. et al. Charge ordering in the rare-earth manganates: the origin of the extraordinary sensitivity to the average radius of the A-site cations. J. Phys. Condens. Matter 10, 8497-8504 (1998).

14. Tokura, Y. \& Tomioka, Y. Colossal magnetoresistive manganites. J. Magn. Magn. Mater. 200, 1-23 (1999).

15. Das, A., Babu, P. D., Chatterjee, S. \& Nigam, A. K. lonic size effect in chargeordered $\mathrm{La}_{0.5} \mathrm{Ca}_{0.5} \mathrm{MnO}_{3}$. Phys. Rev. B 70, 224404 (2004).

16. Mavani, K. R. \& Paulose, P. L. Effects of cation disorder and size on metamagnetism in A-site substituted $\mathrm{Pr}_{0.5} \mathrm{Ca}_{0.5} \mathrm{MnO}_{3}$ system. Appl. Phys. Lett. 86, 162504 (2005).

17. Martin, $\mathrm{C}$. et al. Magnetic phase diagram of Ru-doped $\mathrm{Sm}_{1-x} \mathrm{Ca}_{x} \mathrm{MnO}_{3}$ manganites: Expansion of ferromagnetism and metallicity. Phys. Rev. B 63, 174402 (2001).

18. Mahendiran, R., Raveau, B., Hervieu, M., Michel, C. \& Maignan, A. Instability of metal-insulator transition against thermal cycling in phase separated $\mathrm{Cr}$-doped manganites. Phys. Rev. B 64, 064424 (2001).

19. Pradhan, K. Mukherjee, A. \& Majumdar, P. Distinct effects of homogeneous weak disorder and dilute strong scatterers on phase competition in manganites. Phys. Rev. Lett. 99, 147206 (2007).

20. Das, K., Dasgupta, P., Poddar, A. \& Das, I. Significant enhancement of magnetoresistance with the reduction of particle size in nanometer scale. Sci. Rep. 6, 20351 (2016).

21. Dong, S., Gao, F., Wang, Z. Q., Liu, J. M. \& Ren, Z. F. Surface phase separation in nanosized charge-ordered manganites. Appl. Phys. Lett. 90, 082508 (2007).

22. Das, K., Rawat, R., Satpati, B. \& Das, I. Giant enhancement of magnetoresistance in core-shell ferromagnetic charge ordered nanostructures. Appl. Phys. Lett. 103, 202406 (2013).

23. Zhu, Y. et al. Chemical ordering suppresses large-scale electronic phase separation in doped manganites. Nat. Commun. 7, 11260 (2016).
24. Chen, $\mathrm{L}$. et al. Evolution of the intrinsic electronic phase separation in $\mathrm{La}_{0,6} \mathrm{Er}_{01} \mathrm{Sr}_{03} \mathrm{MnO}_{3}$ perovskite. Sci. Rep. 6, 14 (2016).

25. Chai, X., Xing, H. \& Jin, K. Evolution of photoinduced effects in phase-separated $\mathrm{Sm}_{0.5} \mathrm{Sr}_{0.5} \mathrm{Mn}_{1-y} \mathrm{Cr}_{y} \mathrm{O}_{3}$ thin films. Sci. Rep. 6, 23280 (2016).

26. Shao, J. et al. Emerging single-phase state in small manganite nanodisks. Proc. Natl Acad. Sci. USA 113, 9228-9231 (2016).

27. Zhang, K. et al. Manipulating electronic phase separation in strongly correlated oxides with an ordered array of antidots. Proc. Natl Acad. Sci. USA 112, 9558-9562 (2015).

28. Elovaara, T., Majumdar, S., Huhtinen, H. \& Paturi, P. Photoinduced colossal magnetoresistance under substantially reduced magnetic field. Adv. Funct. Mater. 25, 5030-5037 (2015).

29. Zhang, K. et al. Quantum percolation and magnetic nanodroplet states in electronically phase-separated manganite nanowires. Nano Lett. 17, 1461-1466 (2017).

30. Tokura, Y. Critical features of colossal magnetoresistive manganites. Rep. Prog. Phys. 69, 797-851 (2006).

31. Tomioka, Y., Hiraka, H., Endoh, Y. \& Tokura, Y. Multicritical phase diagram of the electronic states $S_{m 1}-x S_{r} x M n_{O} 3(0.3<x<0.6)$ single crystals with controlled carrier density. Phys. Rev. B 74, 104420 (2006).

32. Gong, G. Q. et al. Colossal magnetoresistance in the antiferromagnetic La0.5Ca0.5MnO3 system. J. Appl. Phys. 79, 4538 (1996).

33. Prellier, W. et al. High magnetic field transport measurements of chargeordered $\mathrm{Pr}_{0.5} \mathrm{Ca}_{0.5} \mathrm{MnO}_{3}$ strained thin films. Phys. Rev. B 66, 24432 (2002).

34. Buzin, E. R. et al. Control of the colossal magnetoresistance by strain effect in $\mathrm{Nd}_{05} \mathrm{Ca}_{0.5} \mathrm{MnO}_{3}$. Thin Films 79, 647 (2001).

35. Rauwel, E., et al Stress deformations and structural quenching in chargeordered $\mathrm{Sm}_{0.5} \mathrm{Ca}_{0.5} \mathrm{MnO}_{3}$. Thin Films 98, 093903 (2005).

36. Ghivelder, L. et al. Abrupt field-induced transition triggered by magnetocaloric effect in phase-separated manganites. Phys. Rev. B 69, 214414 (2004).

37. Tang, T., Huang, R. S. \& Zhang, S. Y. Ultra-sharp metamagnetic transition in manganite $\operatorname{Pr}_{0.6} \mathrm{Na}_{0.4} \mathrm{MnO}_{3}$. J. Magn. Magn. Mater. 321, 263-266 (2009).

38. Mahendiran, R. et al. Ultrasharp magnetization steps in perovskite manganites. Phys. Rev. Lett. 89, 286602 (2002).

39. Rana, D. S., Nirmala, R. \& Malik, S. K. Ultra-sharp metamagnetic transitions in the half-doped manganite compound $\mathrm{Eu}_{0.5} \mathrm{Sr}_{0.5} \mathrm{MnO}_{3}$. Eur. Lett. 70, 376-382 (2005).

40. Kurbakov, A. I. et al. Crystal structure and magnetic and transport properties of $\mathrm{Sm}_{0.5} \mathrm{Sr}_{0.5} \mathrm{MnO}_{3}$ : a -type antiferromagnetic phase and ferromagnetic polarons. Phys. Rev. B 72, 184432 (2005).

41. Kurbakov, A. I., Lazuta, A. V. \& Ryzhov, V. A. Phase diagram of $\mathrm{Sm}_{1-\mathrm{x}} \mathrm{Sr}_{x} \mathrm{MnO}_{3}$ perovskite manganites. J. Phys. Conf. Ser. 200, 012099 (2010).

42. Dagotto, E. Complexity in strongly correlated electronic systems. Science $\mathbf{3 0 9}$ 257-262 (2005)

43. Maji, B., Suresh, K. G. \& Nigam, A. K. Observation of spontaneous magnetization jump and field-induced irreversibility in Nd5Ge3. Europhys. Lett. 91, 37007 (2010).

44. Liao, D., Sun, Y., Yang, R., Li, Q. \& Cheng, Z. Spontaneous magnetization and resistivity steps in the bilayered manganite $\left(\mathrm{La}_{0.5} \mathrm{Nd}_{0.5}\right)_{1.2} \mathrm{Sr}_{1.8} \mathrm{Mn}_{2} \mathrm{O}_{7}$. Phys. Rev. $B$ 74, 174434 (2006).

45. $\mathrm{Wu}$, T. \& Mitchell, J. F. Magnetization steps in manganite films: time delay of the metamagnetic transition. Phys. Rev. B 69, 100405 (2004).

46. Shankaraiah, N., Murthy, K. P. N., Lookman, T. \& Shenoy, S. R. Incubation times and entropy barriers in martensitic kinetics: Monte Carlo quench simulations of strain pseu-dospins. Europhys. Lett. 92, 36002 (2010).

47. Hardy, V. et al. Observation of spontaneous magnetization jumps in manganites. Phys. Rev. B 68, 220402 (2003). R.

48. Dagotto, E., Hotta, T. \& Moreo, A. Colossal magnetoresistant materials: the key role of phase separation. Phys. Rep. 344, 1-153 (2001).

49. Yunoki, S., Moreo, A. \& Dagotto, E. Phase separation induced by orbital degrees of freedom in models for manganites with Jahn-Teller phonons. Phys. Rev. Lett. 81, 5612 (1998).

50. Mukherjee, A., Pradhan, K. \& Majumdar, P. Conductance switching and inhomogeneous field melting in the charge ordered manganites. Europhys. Lett. 86, 27003 (2009).

51. Pradhan, K. \& Yunoki, Y. Nanoclustering phase competition induces the resistivity hump in colossal magnetoresistive manganites. Phys. Rev. B 96, 214416 (2017).

52. Kumar, S. \& Majumdar, P. A travelling cluster approximation for lattice fermions strongly coupled to classical degrees of freedom. Eur. Phys. J. B 50,571-579 (2006). 